\title{
Information and measurement system for determining the acceleration of gravity based on a ballistic gravimeter with a two- dimensional video system
}

\author{
Anna Humeniuk ${ }^{1, *}$, Olena Bezvesilna ${ }^{2}$, Martin Bogdanovskyi ${ }^{1}$, and Valentyn Yanchuk ${ }^{1}$ \\ ${ }^{1}$ Zhytomyr Polytechnic State University, Department of Automation and Computer-Integrated Technologies named after Professor B.B. \\ Samotokin, Zhytomyr, Ukraine \\ ${ }^{2}$ National Technical University of Ukraine "Igor Sikorsky Kyiv Polytechnic Institute”, Instrumentation Department, Kyiv, Ukraine
}

\begin{abstract}
The aim of the sustainable development strategy is to introduce European standards of living in Ukraine and Ukraine's leading position in the world. Active development is observed in information and measurement systems. The article provides a refined mathematical model of the new ballistic laser gravimeter. The composition and structure of the main errors of the ballistic laser gravimeter are given. The main errors of the ballistic laser gravimeter are calculated and estimated. The computer behavior of ballistic laser gravimeter behavior for the most unfavorable resonance modes for different ratios of disturbance values and proper ballistic laser gravimeter parameters is performed. It is suggested to use digital video as a medium of measurement information in the new ballistic laser gravimeter.
\end{abstract}

\section{Introduction}

Today, Ukraine needs to become a state with strong economies and advanced innovations. To do this, first of all, it is necessary to restore macroeconomic stability, to ensure sustainable economic growth in an environmentally sustainable way, to create favorable conditions for economic activity. Active development is also observed in information and measurement systems. Modern systems must meet both the technical and environmental requirements of humanity.

Measuring the absolute value of gravity acceleration with high accuracy is necessary for solving a wide range of scientific problems: determining the shape of the Earth, constructing models of motion of deep masses, predicting earthquakes, inventing deep density inhomogeneities, finding minerals, and others. Gravimeters are used to determine $g$. Among them, the most well-known for terrestrial measurements are ballistic laser gravimeters (BLG).

It is known that the use of computers as simulation devices can: reduce, and in some cases completely eliminate the need for physical experiments with real devices, greatly reduce the time, improve productivity, accuracy of research all this has a significant economic effect.

The article [1] describes an advanced gravimetric system for low flow rates that was developed by the National Institute of Metrology of Japan (NMIJ) to perform the method of flight-start and stop calibration. However, there is no description of the mathematical model.
The paper [2] presents the regional gravimetric survey of the central part of the Republic of Slovenia. The need for a new gravimetric survey, the survey plan and the actual field measurements are presented. Data processing, control calculations, data adjustment in the form of a gravimetric network and accuracy estimations of the results obtained during the regional gravimetric survey are described.

Today new information-measuring gravimetric systems, methods and tools of gravimetric measurements are being developed by the Ukrainian scientists of National Technical University of Ukraine "Igor Sikorsky Kyiv Polytechnic Institute", Zhytomyr Polytechnic State University [3].

But there is no information in the literature regarding the mathematical model of BLG that is required for further computer modeling. In this regard, the analysis of the device, the study of the main errors affecting the measurement is an urgent task.

The task of measuring gravity by ballistic methods is to measure length and time. This follows, for example, from the analysis of the dimension of acceleration. Therefore, the mathematical model should reveal the analytical relation of the path traveled by the test body with time and external influences. The most constructive approach would be to build a model in which, on the one hand, the free motion of the test body in the inertial coordinate system is considered, taking into account the vertical gradient of the gravity acceleration and the forces of resistance, and on the other, the law of motion of some coupled coordinate system is shifted under the influence of external inertial influences and holds the reference system of the gravimeter.

* Corresponding author: gum ann@ukr.net 
Considering all researches, it can be concluded that the current scientific and technical problem today is to improve the accuracy of measurement of the gravity acceleration.

The purpose is to get a mathematical model of the new BLG and improve the measurement accuracy of the gravity acceleration.

\section{Suggestions and solutions}

Consideration of the free motion of a test body tilted vertically upwards in an inertial coordinate system is reduced to solving a nonlinear 2 nd order differential equation of the following form:

$$
m \cdot \ddot{z}=m \cdot\left(g_{0}+\alpha \cdot z\right)-\gamma_{1} \cdot \dot{z}-\gamma_{2} \cdot(\dot{z})^{2},
$$

where $m$ is the mass of the test body; $z$ is the vertical coordinate; $\alpha$ is the vertical gradient; $\gamma_{1}, \gamma_{2}$ are the coefficients that determine the contribution of resistance forces proportional to the first and second degrees of speed of motion of the test body.

Solving equation (1) by successive approximation using the Laplace transform and then decomposing it by $z$, leads to the following equation:

$$
z(t)=g_{0} \cdot \sum_{n=0}^{\infty} A_{n} \cdot t^{n},
$$

where $A_{n}$ is the set of coefficients $(n=0,1,2, \ldots)$ determined from the conditions of motion of the test body in the ballistic block relative to the reference system.

This expression describes the motion of the test body in the inertial coordinate system. The coordinates of a test body in real-world measurements of the gravity acceleration is determined by the fixed coordinate system that exposed perturbation an inertial system are uniquely dependent on its coordinates in the connected system:

$$
\bar{R}_{i}=\bar{r}+\bar{R}
$$

where $\bar{R}_{i}$ - a radius vector of the test body in the inertial system;

$\bar{r}-$ a radius vector of the test body in the connected system;

$\bar{R}$ - a radius vector that describes the offset of a connected system.

Generally, the ballistic gravity meter is operated so that the sensitivity axis (in our case the $z$ axis) is held vertically. Therefore, equation (3) can be simplified:

$$
S(t)=z(t)+R_{z}(t)
$$

In this expression, the component $S(t)$ describes the behavior of the test body in the inertial system, and component $R_{\mathrm{z}}(t)$ - the influence of external perturbations.

Algorithms for measurements of the gravity acceleration using the described model are very diverse, both by the method of realization of free movement of the test mass and by methods of measuring the path and time.
Depending on the method of realization of the free movement of the test body, all terrestrial methods of determining $g$ can be divided into two groups

- with asymmetrical free movement;

- with symmetrical free movement.

Using symmetric methods (both branches of the parabola in Fig. 1), the test body is tilted up, and the path and time measurements are made on the left and right branches of the trajectory.

When using asymmetric methods (right branch of the parabola in Fig. 1), the test body moves freely (falls) in the vacuum.

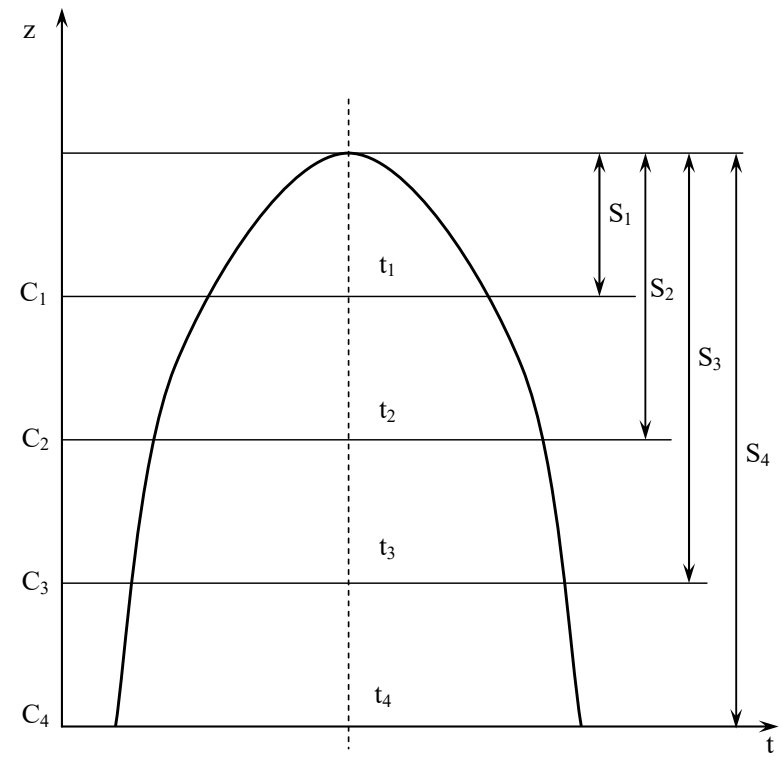

Fig. 1. The flight path of the test body in ballistic gravimeter: $C_{1}-C_{4}-$ observation levels; $t_{1}-t_{4}-$ moments of end of measurement intervals; $S_{1}-S_{4}$ - measurement intervals.

These methods are distinguished by the relatively simple implementation of free movement and the absence of the need to apply impulse (shock) force when starting the test body, which depends on the height of the throw, which greatly improves the dynamic conditions of gravimeter $[4,5]$.

The advantages of symmetric methods include the possibility of virtually complete elimination of systematic errors (which is proportional to the first degree of speed of motion of the test body) with a relatively simple measurement algorithm, as well as the possibility of reducing the vertical dimensions of the device, because at the same height of the gravimeter is the total path traveled by the body in this case it will be big. However, at the moment of throw there is a pulse reactive force, which introduces an error in the measurement results.

Now we will present the set and structure, calculate and evaluate the main errors of BLG.

A further increase in accuracy is hampered by a number of circumstances, both of a fundamental (physical) and technological (economic) nature. Therefore, there is the question of optimal accuracy, which depends on many factors and is determined by limitations. These limitations need to be known so that, on the one hand, you can use all of the precise possibilities presented by the theory, and on 
the other hand, do not make unsuccessful attempts to increase accuracy beyond what is possible.

Let us analyze how practical considerations shape the conditions for the required accuracy of measurements in gravimetry.

Let us take a slow (quasi-static) process. It is necessary with the given reliability $\beta$ to obtain their value $\Delta x(t)$ (Fig. 2), measuring the process $x(t)$ over time $\tau$.

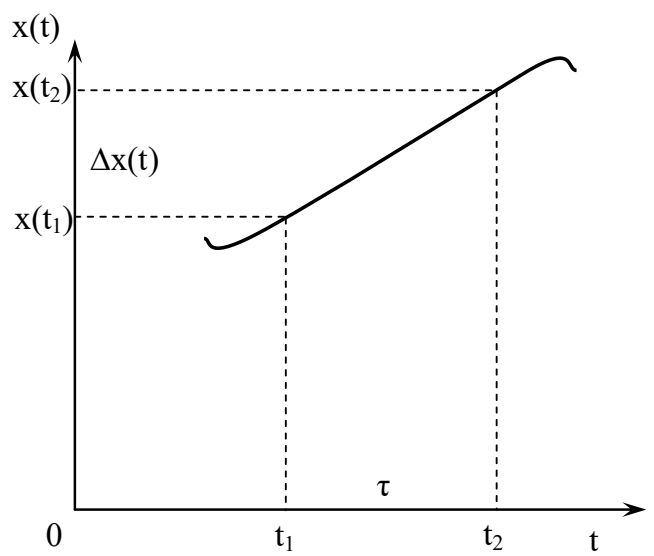

Fig. 2. The dependence of the permissible error on the drift velocity of the measurement process: $S(t)$ - change of learning parameter of process during observation $\tau$.

The magnitude measured by $\Delta x(t)$ and the error $\delta(t)$ of BLG are related by the relation:

$$
\Delta x(t) \geq \beta \cdot \delta(t),
$$

where $\beta \geq 1$ is the number that characterizes the reliability of the measurement.

Then the Lagrange theorem on the mean in the interval under consideration can be written as follows:

$$
\frac{x\left(t_{2}\right)-x\left(t_{1}\right)}{t_{2}-t_{1}}=\frac{\Delta x(t)}{\tau}=\frac{d x}{d t}=V(t),
$$

whence, taking into account equation (5), we obtain:

$$
\delta(t) \leq \tau \cdot \frac{V(t)}{\beta}
$$

From equation (6) it follows that in the case of measurements of slowly changing processes, in addition to trivial to the accuracy of the measuring instrument, the requirements are also not completely obvious (measurement triviality is contained in the fact that the error of the device $\delta(t)$ must be smaller the more reliable the result is):

1) the error value of the instrument must change over time if the process speed $V(t)$ is variable;

2 ) the value of the error of the instrument must be less than the smaller time interval $\tau$ spent to detect changes $\Delta S$.

Based on equation (6), we determine the maximum permissible value of $\delta(t)$.

If $\tau=0,1 ; \beta=1 ; V=1 \mu \mathrm{Gal}$ then $\delta=\frac{0,1 \cdot 1}{1}=0,1 \mu \mathrm{Gal}$ or in relative terms $\delta / g_{0}=1 \cdot 10^{-10}$.

Such an error in the measurement of the gravity acceleration is currently needed to solve some geological problems [6]. Approximately the same accuracy order is required by an exemplary gravimeter for calibration of accelerometers.

But this accuracy, at this point in time, is close to the limits recognized by some instrumental and fundamental limitations.

As is known, the error of the laser interferometer, which is a device for reproducing a unit of length, has a significant effect on the error of the gravimeter. Let us define one of the errors of the interferometer - the diffraction error caused by the limited aperture of the light beam:

$$
\frac{\Delta g}{g} \approx 0,1 \cdot\left(\frac{\lambda}{d}\right)^{2}
$$

At wave length $\lambda=0,633 \mu \mathrm{m}$; beam diameter $d \approx 2 \mathrm{~mm}$ : $\frac{\Delta g}{g}=0,1 \cdot\left(0,633 \cdot \frac{10^{-6}}{2} \cdot 10^{-3}\right)^{2} \approx 1,0017225 \cdot 10^{-8}$.

In modern gravimetry, the total interaction of gravitational obstacles, with the exception of the influence of the sun and the moon, is considered so small in comparison with the equilibrium of inertial forces that it can be neglected [7]. The very exclusion of the influence of inertial forces on the results of measuring the gravimeter is, at this point in time, one of the main difficulties in improving the accuracy of the determination of the gravity acceleration.

When performing gravimetric measurements of higher accuracy, a number of systematic errors are taken into account by making appropriate corrections, such as light pressure corrections, first and second order vertical and horizontal gradients, vacuum resistance, etc.

Although accurate accounting for these errors and corrections is now more difficult than measuring acceleration itself, it is fundamentally feasible. However, there are fundamental limitations that cannot be eliminated by any tools or technological means. These include the approximation of the set value of the speed of light, quantum-mechanical constraints, the limits of the accuracy of determining the acceleration gradients, fluctuations, etc.

Limitations that impose quantum mechanical laws:

$$
(\Delta \bar{E})^{2} \cdot(\Delta \bar{t})^{2} \geq h^{2}
$$

This inequality imposes a restriction on the measurement of energy (velocity) of the body if it is to be measured at a precise time. As noted above, the acceleration $\mathrm{g}$ can be determined from the relation:

$$
g=\frac{V-V_{0}}{t-t_{0}} .
$$

Find the variance of the gravity acceleration:

$$
\begin{gathered}
D=M_{2}\{g\}=\frac{g^{2}}{\left(t-t_{0}\right)^{2}} \cdot\left[(\Delta \bar{t})^{2}+\left(\Delta \bar{t}_{0}\right)^{2}\right]+ \\
+\frac{1}{\left(t-t_{0}\right)^{2}} \cdot\left[(\Delta \bar{V})^{2}+\left(\Delta \bar{V}_{0}\right)^{2}\right],
\end{gathered}
$$


where $(\Delta \bar{t})^{2}$ and $\left(\Delta \bar{t}_{0}\right)^{2}$ - the variance (uncertainty) of the end and start points of time; $(\Delta \bar{V})^{2}$ and $\left(\Delta \bar{V}_{0}\right)^{2}-$ dispersions of the finite and initial velocities of the freefall body.

Rewrite equation (8) using equation (7):

$$
\begin{gathered}
D=\frac{g^{2}}{\left(t-t_{0}\right)^{2}} \cdot\left[(\Delta \bar{t})^{2}+\left(\Delta \bar{t}_{0}\right)^{2}\right]+ \\
+\frac{h^{2}}{m^{2}\left(t-t_{0}\right)^{2}} \cdot\left[\frac{1}{V^{2}(\Delta \bar{t})^{2}}+\frac{1}{V_{0}^{2}\left(\Delta \bar{t}_{0}\right)^{2}}\right],
\end{gathered}
$$

Investigating equation (9) for the extremum, let us determine $(\Delta \bar{t})^{2}$ and $\left(\Delta \bar{t}_{0}\right)^{2}$, that correspond to the minimum $D$

$$
(\Delta \bar{t})^{2}=\frac{h}{m V g} \text { and }\left(\Delta \bar{t}_{0}\right)^{2}=\frac{h}{m V_{0} g} .
$$

So, we see that measuring of the gravity acceleration more accurately than $10^{-9}$ is impossible in principle due to quantum mechanical constraints, as well as fluctuations in length and time measures.

After further analysis of the literature, it can be seen that the error caused by the linear drift of the scale factor of the BLG is $\Delta_{l}=0.002 "$.

The error value is insignificant, so it is possible to neglect such a linear drift of the scale factor.

The error from the portable (relatively BLG) angular velocity of the Earth rotation is determined by the formula:

$$
\Delta_{E}=\frac{\omega_{z}}{k}
$$

where $k$ is the transmission coefficient of BLG, $\omega_{\mathrm{z}}$. is the vertical component of the portable angular velocity of the Earth rotation.

Note that the vertical component of the portable angular velocity of the Earth rotation is:

$$
\omega_{z}=\omega_{E} \sin \phi
$$

Find the numerical value $\Delta_{E}$ for such parameters $k=5 \cdot 10^{-3} \mathrm{~kg} \cdot \mathrm{m}, \omega_{E}=7,29 \cdot 10^{-5} \mathrm{~s}^{-1}$.

The maximum value of the term $\frac{\omega_{E} \sin \phi}{k}$ corresponding to $\varphi=90^{\circ}$ is $1,46 \cdot 10^{-8} \mathrm{rad}$. That is, the error value $\triangle_{E}$ is small and can also be neglected.

The possibility of increasing the accuracy and speed of a ballistic gravimeter by determining the influence of the deviation of the gravity axis of the gravimeter from the local vertical direction by using digital video has been investigated [8].

The problem is solved by the fact that in the already existing gravimetric system with high-precision alignment of the axis of gravity of the gravimeter is additionally introduced video camera 5 , the processor of linear approximation of the label 6 , the reflecting element 7 , the photoelectric autocollimator 8 , and the body of the gravimeter 1 is plotted, the direction of which coincides with the direction of the axis of gravity of the gravimeter 1 , and the label is optically connected to the input of the camcorder 5, the output of which is connected to the input of the processor 6 linear approximation of the label, the output of which is connected to the first input of the digital computer 3, the second input of which is connected to the output of the photoelectric autocollimator 8 , the input of which is optically connected to the reflecting element 7 , which is attached to the housing gravimeter 1 (Fig. 3).

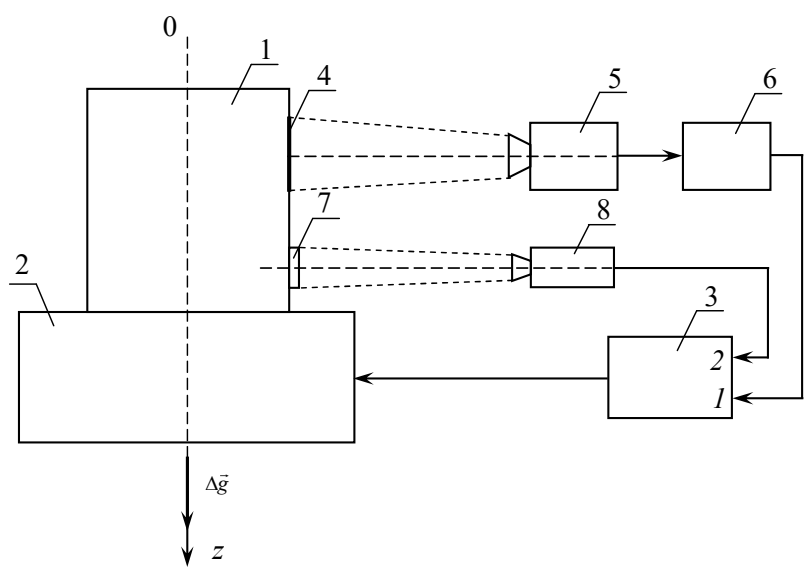

Fig. 3. Schematic diagram of a gravimetric system with high precision alignment of the axis of sensitivity of the gravimeter.

High-precision alignment of the axis of sensitivity of the gravimeter in three-dimensional space is ensured by the high-precision exposure in the three-dimensional space of the position of the plane corresponding to the surface of the reflecting element. Moreover, the mark and the reflecting element are fixed on the body of the gravimeter so that the direction of the mark coincides with the direction of the axis of sensitivity of the gravimeter, and the surface plane of the reflecting element was parallel to that axis of sensitivity [9].

The photoelectric autocollimator and camcorder are also pre-oriented in three-dimensional space so that their optical axes are perpendicular to the local vertical direction. With the help of a photoelectric auto-collimator, the deviation of the surface plane of the reflecting element from the position when it is perpendicular to the optical axis of the auto-collimator is estimated. A signal proportional to the degree of this mismatch is fed to the computer. The computer manages the spatial position of the platform in such a way so to eliminate this mismatch.

The result is an arrangement of the axis of sensitivity of the gravimeter in a vertical plane that is perpendicular to the optical axis of the photoelectric autocollimator and in which the direction of the local vertical is located.

However, the gravity axis of the gravimeter may be located in the specified vertical plane, but deviate some angle from the direction of the local vertical. This deviation can be determined by the tag, camcorder, and linear tag approximation processor. With the help of a digital computer that manages the spatial position of the platform, this deviation can be eliminated.

The result is a highly accurate alignment of the gravity axis of the gravimeter in three-dimensional space and, accordingly, its high-precision coincidence with the direction of the local vertical and the full vector of gravity acceleration.

Thus, in the gravimetric system, the accuracy of measuring the acceleration of the force of gravity is significantly increased. 


\section{Simulations}

The problem of investigating the influence of disturbance action parameters and some own BLG parameters on the work with the help of a computer is solved. form:

We use the equation of motion BLG, writing it in the

$$
\begin{gathered}
\ddot{\alpha}+\dot{\alpha}[2 n-L \sin (\omega t+\varepsilon)]+\omega_{0}^{2} \alpha= \\
=N \sin \omega t,
\end{gathered}
$$

where $\quad L=\frac{c_{1}^{\prime}}{H^{2}} m l w_{b}, N=\frac{m l k_{1}}{H^{2}} w_{a} \quad-\quad$ vibration parameters.

Given $M(t)=2 n-L \sin (\omega t+\varepsilon), D(t)=\omega_{0}^{2}$, then

$$
\ddot{\alpha}+\dot{\alpha} \cdot M(t)+D(t) \cdot \alpha=0,
$$

where $M(t)$ and $D(t)$ are $T$-periodic functions, $\dot{M}(t)$ and $\dot{D}(t)$ assume integrated piecewise-continuous.

The equation of the form (11) can be reduced to a similar one without changing the characteristic indices, where $M(t)=$ const.

$$
\int_{0}^{t} M\left(t_{1}\right) d t_{1}=\kappa t+M_{1}(t)
$$

where

$$
\aleph=2 n ; M\left(t_{1}\right)=\int_{0}^{t}\left[M\left(t_{1}\right)-\aleph\right] d t=\frac{L}{\omega} \cos (\omega t+\varepsilon),
$$

and $M_{1}(t)$ is $T$-periodic function.

Replacing

$$
\alpha=e^{-\frac{1}{2} M_{1}(t)} x=e^{-\frac{1 L}{2 \omega} \cos (\omega t+\varepsilon)} x,
$$

we get

where

$$
\ddot{x}+2 n \dot{x}+F(t) x=0,
$$

$$
\begin{aligned}
F(t) & =D(t)-\frac{1}{4} M^{2}(t)-\frac{1}{2} \dot{M}(t)+\frac{1}{4} \aleph^{2}= \\
& =\omega_{0}^{2}+v_{0} \sin \left(\omega t+\varepsilon+\sigma_{8}\right),
\end{aligned}
$$

where $\sigma_{8}=\operatorname{arctg} \frac{\omega}{2 n}, v_{0}=\frac{L \sqrt{\omega+4 n^{2}}}{2}$.

Given (5.1) and (5.5), expression (5.4) can be written as

$\ddot{x}+2 n \dot{x}+\left[\omega_{0}^{2}+v_{0} \sin \left(\omega t+\varepsilon+\sigma_{8}\right)\right] x=N \sin \omega t$

or, taking into account the real BLG parameters:

$\ddot{x}+2 \xi \omega_{0} \dot{x}+\left(\omega_{0}^{2}+v_{1} w_{b} \sin \omega t\right) x=0,625 w_{a} \sin \omega t$,

where $v_{1}=\frac{v_{0}}{w_{b}}$.

Therefore, the BLG motion equation (10) is transformed into one equation (16), which is convenient for computer simulation. The equation obtained is a Mathieu-Hill equation [10-12].
The graphs of some functional dependences for certain values $w_{a}, w_{b} \omega$, as well as the values of the damping coefficient $\xi$, are shown in Fig. 4, 5, 6.

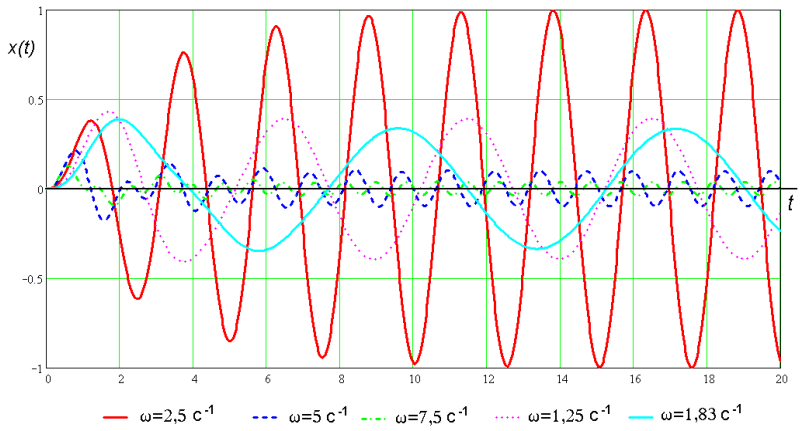

Fig. 4. Graphs of change of output signal for different values $\omega$.

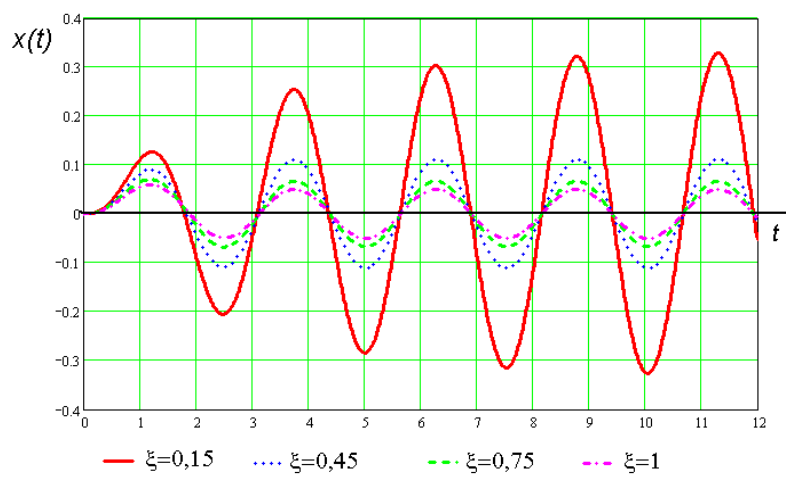

Fig. 5. Graphs of change of the output signal $x(t)$ for different values of the damping factor $\xi$.

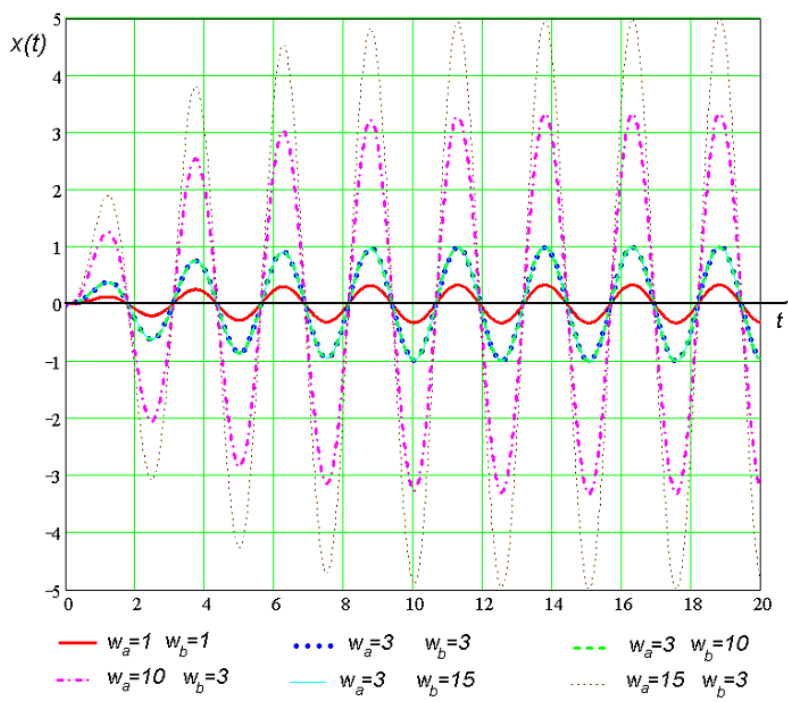

Fig. 6. Graphs of output signal $x(t)$ changes for different values $w_{a}, w_{b}$.

The graphs show that:

- at perturbation frequency $\omega=\omega_{0}=2,5 \mathrm{~s}^{-1}$ the main resonance occurs, the most dangerous for the $\mathrm{BLG}$;

- at perturbation frequency $\omega=\omega_{0} / 2=1,25 \mathrm{~s}^{-1}$, $\omega=\omega_{0} / 3=0,83 \mathrm{~s}^{-1}$ the output signal is not distorted (sub harmonic oscillations are set); 
- at perturbation frequencies $\omega=2 \omega_{0}=5 \mathrm{~s}^{-1}$, $\omega=3 \omega_{0}=7,5 \mathrm{~s}^{-1}$ the output signal is distorted (beating is set);

- increasing the amplitudes of horizontal accelerations does not affect the amplitude of the forced oscillations of the BLG $x(t)$;

- the amplitudes of the forced oscillations along the axis of the BLG sensitivity are directly proportional to the amplitudes of the perturbing vibration accelerations along the axis of the BLG sensitivity.

In order to compare the above information, the results of modeling the equations of motion of $\mathrm{BG}$ on the computer are summarized in Table 1.

Table 1. The amplitudes of the steady forced oscillations of the BLG.

\begin{tabular}{|c|c|}
\hline $\boldsymbol{\omega}, \mathbf{s}^{-1}$ & $\alpha, \mathbf{r a d}$ \\
\hline 0,83 & $0,22 \cdot 10^{-7}$ \\
1,25 & $0,65 \cdot 10^{-7}$ \\
2,5 & $1,86 \cdot 10^{-6}$ \\
5,0 & $0,93 \cdot 10^{-6}$ \\
7,5 & $4,7 \cdot 10^{-7}$ \\
\hline
\end{tabular}

Comparing the amplitudes of the steady forced oscillations of the BLG at $\omega=\omega_{0}, 3 \omega=\omega_{0}, 2 \omega=\omega_{0}$, $\omega=2 \omega_{0}, \omega=3 \omega_{0} ; \xi=1 ; w_{a}=w_{b}=1 \mathrm{~m} / \mathrm{s}^{2}$, calculated in accordance with expression (12) (Table 1), it is seen that the amplitudes of the steady forced oscillations of the BLG are greatest, provided that the frequency of the natural oscillations of the BLG and the disturbing influence are equal.

Digital simulation of the effect of perturbation parameters on the BLG, as well as the eigen parameters, confirmed the main advantage of the BLG over the known gravimeters - its higher accuracy (mean square error of $0,1 \mu \mathrm{Gal})$.

\section{Conclusions}

A refined mathematical model of the new BLG was obtained. The composition and structure of the main errors of the BLG are given. The main errors of the BLG are calculated and estimated. The computer simulation of BLG behavior for the most unfavorable resonance modes for different ratios of disturbing factors and intrinsic BLG parameters is performed. The conclusions made in the analytical study of the work of BLG are confirmed: the most dangerous from the point of view of resonance occurrence is only the case of coincidence of perturbation frequency with the frequency of natural BLG oscillations. As the damping factor increases, the resonance disappears. The main advantage of BLG over the known gravimeters was confirmed - its greater accuracy (mean square error of $0.1 \mu \mathrm{Gal}$ ). It is proposed to use digital video in the new BLG as a carrier of measurement information. This extends the functionality and also significantly improves the accuracy of measurements of the gravity acceleration. This reflects the modern engineering and technological solutions of the information technology era in the sustainable development of society.

\section{References}

1. K.-H. Cheong, R. Doihara, T. Shimada, Y. Terao, Flow Measurement and Instrumentation 56, 1-13 (2017). doi:10.1016/j.flowmeasinst.2017.05.006

2. K.K. Medveda, M. Kuharb, B. Kolerb, Measurement 136 395-404

(2019). doi:10.1016/j.measurement.2018.12.065

3. O. Bezvesilna, A. Tkachuk, A. Humeniuk, S. Nechai, Rozrakhunok ta analiz statychnykh pokhybok dvohiroskopnoho chutlyvoho elementa (Calculation and analysis of static errors of two-gyro sensor). Technology audit and production reserves 6/2(32), 9-12 (2016). doi:10.15587/23128372.2016.85452

4. I.A. Bunin, E.N. Kalish, D.A. Nosov, M.G. Smirnov, Y.F. Stus, Optoelectronics Instrumentation and data processing 46(5), 476-482 (2007). doi:10.3103/s8756699011050104

5. A. Kaufman, R. Hansen, Methods in Geochemistry and Geophysics 41, 161-215 (2007). doi:10.1016/S0076-6895(07)41004-6

6. I. Korobiichuk, Measurement 89, 151-158 (2016). doi:10.1016/j.measurement.2016.04.017

7. V.Y. Timofeev, E.N. Kalish, D.G. Ardyukov, M.G. Valitov, A.V. Timofeev, Y.F. Stus, R.G. Kulinich, D.A. Nosov, I.S. Sizikov, B. Ducarme, Geodesy and Geodynamics 8, 193-200 (2017). doi:10.1016/j.geog.2017.03.011

8. W. Bich, G. D’Agostino, A. Germak, Reconstruction of the free-falling body trajectory in a rise-and-fall absolute ballistic gravimeter. Metrologia 45, 308312 (2008). doi:10.1088/0026-1394/45/3/007

9. S. Merlet, Q. Bodart, N. Malossi, A. Landragin, F. Pereira Dos Santos, O. Gitlein, L. Timmen, Comparison between two mobile absolute gravimeters: optical versus atomic gravimeters. Metrologia 47, 9-11 (2010). doi:10.1088/0026$1394 / 47 / 4 / 101$

10. J. Arlt, G. Birkl, E. Rasel, W. Ertmer, Atom optics, guided atoms, and atom interferometry. Advances in Atomic, Molecular, and Optical Physics 50, 55-89 (2005). doi:10.1016/s1049-250x(05)80007-2

11. A. Kurin, The Cauchy problem for the Mathieu equation far from parametric resonance. Comput. Math. and Math. Physics 51, 1325 (2011). doi:10.1134/s0965542511080136

12. E.G. Belomytseva, A.F. Kurin, E.B. Tulenko Zadacha Koshi dlya uravneniya Mat'e s zatuhaniem pri parametricheskom rezonanse (The Cauchy problem for the Mathieu equation with damping at parametric resonance). Bulletin of the Voronezh State University. Series: Physics. Maths, 3, 105-125 (2018),

http://www.vestnik.vsu.ru/pdf/physmath/2018/03/20 18-03-09.pdf. Accessed 23 Feb 2020 